\title{
Predicting Human Responses to Syllogism Tasks Following the Principle of Maximum Entropy ${ }^{\dagger}$
}

\author{
Marco Wilhelm and Gabriele Kern-Isberner \\ TU Dortmund, Dortmund, Germany \\ + Presented at the Entropy 2021: The Scientific Tool of the 21st Century, 5-7 May 2021; Available online: \\ https://sciforum.net/conference/Entropy2021/.
}

Published: 5 May 2021

Syllogistic reasoning is one of the major research domains in cognitive science. Syllogisms are quantified semi-logical statements that consist of two premises, each relating two terms by one quantifier out of "All", "No", "Some", and "Some not". While one of the terms is mentioned in both premises, one is interested in what conclusion can be drawn about the relationship between the other two terms. For example, a well-formed syllogism task is "If all A are B and no B is a C, what, if anything, follows about the relationship between A and C?" While some syllogism tasks have a logically valid conclusion (in the example above, "No A is a C." is logically valid), some have not, like "If all A are B and some B are C, what follows about A and C?" In cognitive science, human responses to syllogism tasks are studied in order to better understand the human understanding of quantification and uncertainty in reasoning.

In order to predict human responses to syllogism tasks, we develop a probabilistic model of syllogisms based on the principle of maximum entropy. For this, we translate the premises of syllogisms into probabilistic conditional statements and derive the probability distribution that satisfies the conditional probabilities while having maximal entropy. Then, we calculate the probabilities of all possible conclusions and compare them with the respective quantifier. As a prediction, we basically choose the option with the best matching. Based on empirical data, we show that our maximum entropy model predicts human responses better than established cognitive models.

(C) 2021 by the authors. Licensee MDPI, Basel, Switzerland. This article is an open access article distributed under the terms and conditions of the Creative Commons Attribution (CC BY) license (http://creativecommons.org/licenses/by/4.0/). 